\title{
Arrhythmias in two patients with left ventricular bypass transplants ${ }^{1}$
}

\author{
Brian M. Kennelly, Peter Corte, Jacques Losman, and Christiaan N. Barnard \\ From the Cardiac Clinic and Department of Cardiothoracic Surgery, Groote Schuur Hospital and the \\ Departments of Medicine and Surgery, University of Cape Town, South Africa
}

Two patients who underzent left ventricular bypass transplants are described. Both patients sustained postoperative rhythm disturbances of their own hearts during sinus rhythm of the donor hearts. Illustrative examples of atrial flutter, ventricular flutter, ventricular fibrillation, blocked atrial extrasystoles, and double ventricular parasystole in the recipient hearts are presented. The patients tolerated all these arrhythmias well during uninterrupted sinus rhythm in the donor heart. The problems in interpretation of arrhythmias in the presence of two hearts are discussed.

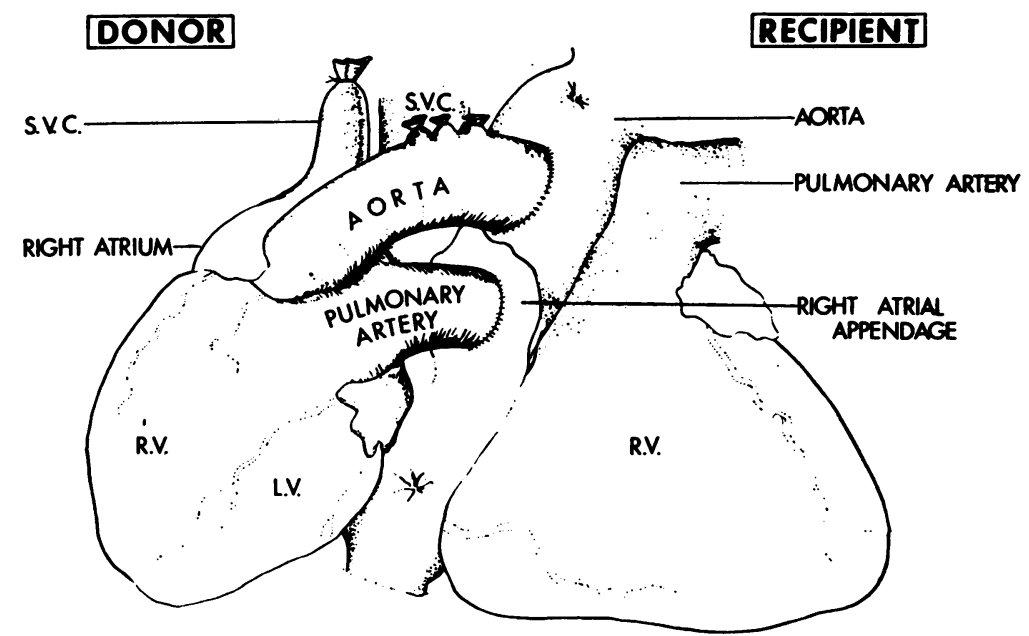

FIG. 1 Position of donor and recipient hearts in anteroposterior view with relevant anastamoses (Barnard and Losman (1975). Reprinted with permission of South African Medical Journal).

The purpose of this report is to illustrate some of the bizarre electrocardiograms resulting from cardiac transplantation in man where the recipient heart is left in situ. The surgical technique was described in detail by Barnard and Losman (1975). Briefly, the donor heart is implanted via a median sternotomy in the area anterior to the hilum of the Received 24 December, 1975.

'Supported by the South African Medical Research Council, the Cape Town City Council, and the Chris Barnard Fund. right lung and in the right cardiophrenic angle. Both venae cavae of the donor heart are ligated and the donor right ventricle pumps only its coronary sinus venous return into the recipient's right atrium via the donor pulmonary artery (Fig. 1). The donor and recipient left atria are anastomosed side-to-side and the donor left ventricle is connected via ita aorta to the recipient aorta.

The sinus nodes of both hearts are intact, but that of the donor is denervated. Clearly, the 
rhythms of both hearts are totally independent and are subject to their own individual disturbances of rhythm. As will be shown, these are usually readily identified where one of the two hearts remains in sinus rhythm. The theoretical possibility exists, however, that the development of arrhythmias in both hearts simultaneously could give rise to considerable diagnostic difficulties.

\section{Case reports}

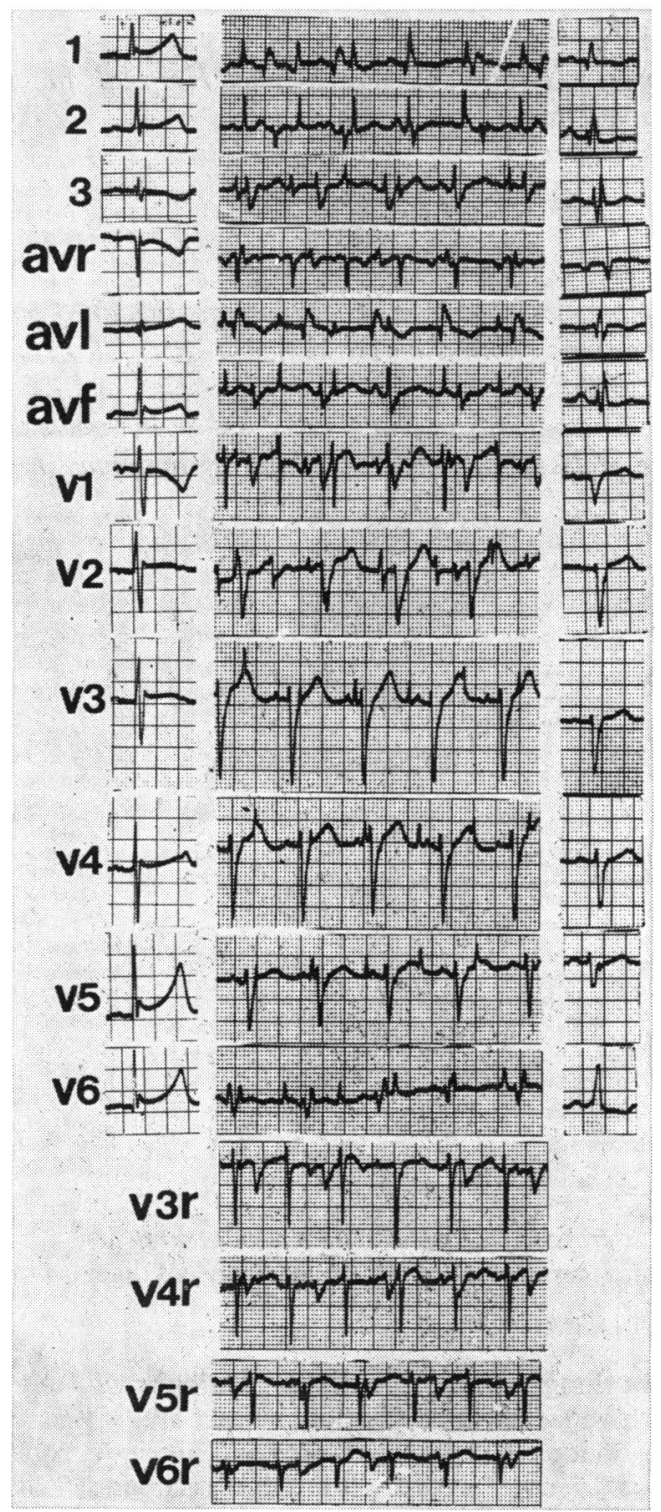

FIG. 2 Case 1: Donor electrocardiogram on left, recipient electrocardiogram on right, and postoperative electrocardiogram showing both donor and recipient complexes in centre panel. Leads V1, V2, and V3 are half-standard on the recipient electrocardicgran.

\section{Case 1}

This 59-year-old man had a 10-year history of intractable ischaemic heart disease progressing to grade 4 effort intolerance. He had clinical evidence of biventricular failure, massive cardiomegaly, a third heart sound, and an electrocardiogram illustrated in the right-hand panel of Fig. 2 showing sinus rhythm with left atrial enlargement and left bundle-branch block. Chest $x$-ray film showed massive cardiomegaly with probable left ventricular aneurysm. Cardiac catheterization showed severe biventricular cardiac failure with significant pulmonary hypertension, and left ventricular angiography revealed extensive destruction of the left ventricular muscle with a chamber which was dilated and contracted very poorly; coronary angiography showed severe three-vessel disease.

In view of his severe symptoms and his unsuitability for a more conservative operation, transplantation of the type described above was recommended to circumvent the problem of his pulmonary hypertension which would otherwise have been a relative contraindication to conventional cardiac transplantation. At the time of operation there was diffuse fibrosis of the left ventricle of which about 20 per cent was excised; there was fresh clot in the left ventricular cavity. The donor heart was obtained from a 10 -year-old girl who died of head injuries. Her electrocardiogram before death is illustrated in the left-hand panel of Fig. 2, showing sinus rhythm with pronounced $J$ wave and prolongation of QT interval resulting from cerebral damage.

The centre panel of Fig. 2 illustrates the electrocardiogram obtained postoperatively showing both donor and recipient hearts in sinus rhythm. The recipient QRS complexes are readily identified by the presence of significant QRS widening as seen before operation. The frontal plane QRS axis of the donor heart is unchanged after transplantation. The frontal plane QRS axis of the patient's own heart has shifted from $+75^{\circ}$ before to $-45^{\circ}$ after operation. The horizontal plane QRS axis of the donor heart has undergone slight counter-clockwise rotation.

Fig. 3 illustrates the development of atrial flutter with 4:1 atrioventricular block on the 19 th postoperative day at a time when the patient was fully 
digitalized. The flutter rate of the recipient heart is an exact multiple of the recipient ventricular rate and not of the donor ventricular rate, thus indicating that the atrial flutter originates in the recipient heart. This rhythm did not cause any haemodynamic disturbance and subsided spontaneously.

On the 22nd postoperative day the patient's condition deteriorated dramatically. He became dyspnoeic and restless with signs of poor peripheral perfusion and raised jugular venous pressure. Whereas previously both donor and recipient heart pulses had been palpable at the wrist, a single pulse wave only could now be palpated. Heart sounds were no longer audible over his own heart but could still be heard over the donor heart. Fig. 4 illustrates the electrocardiogram at this time. A regular ventricular rhythm of 138 /minute is seen, with a QRS morphology of the donor heart. Ventricular fibrillation is clearly present in the recipient heart; this responded promptly to DC countershock synchronized on the QRS complex of the donor heart.

Fig. 5 illustrates the development of ventricular flutter four months after operation in the same patient at a time when he had been readmitted to hospital for observation because of a respiratory infection. In many of the leads it is impossible to determine which heart is in ventricular flutter. However, with knowledge of the previous morphology of the QRS complexes of the two hearts in each lead, the donor heart complexes can be clearly identified in lead aVR at a rate of $170 /$ minute,

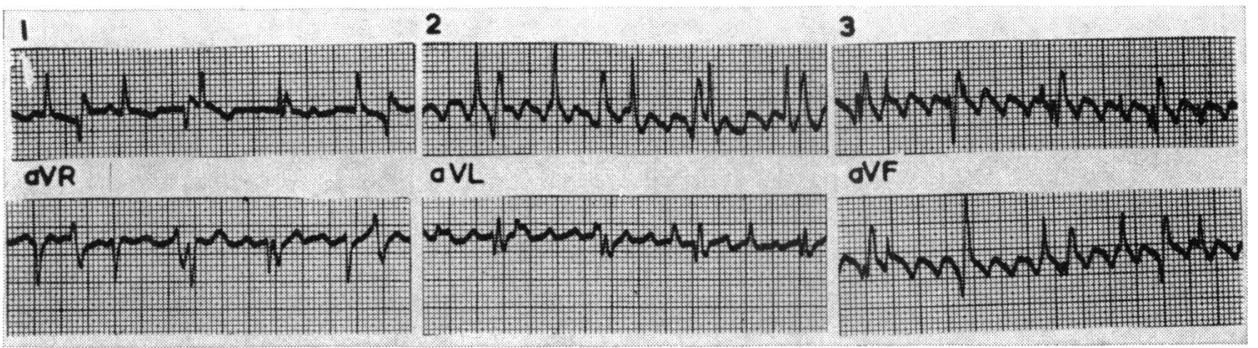

FIG. 3 Atrial flutter with 4:1 AV block in recipient heart of Case 1.

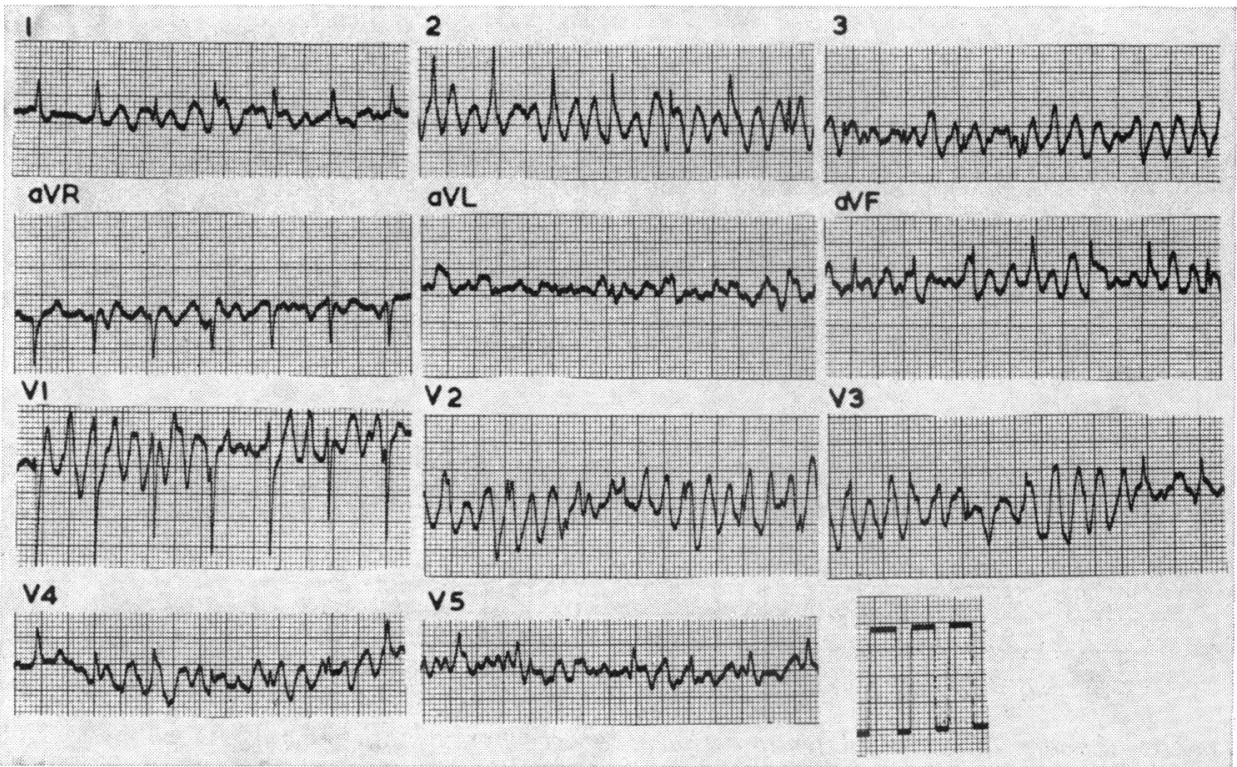

FIG. 4 Ventricular fibrillation in recipient heart of Case 1. 
indicating that the recipient heart is the source of the arrhythmia. On this occasion his clinical condition was as it had been when he was in ventricular fibrillation previously. The arrhythmia responded readily to intravenous lignocaine.

Ventricular arrhythmias were prominent throughout his postoperative course and frequent multiform ventricular extrasystoles are readily seen in Fig. 6. The same record shows occasional 'dropped beats' in the recipient heart rhythm, the explanation of which is not obvious. The recipient $R R$ interval is $600 \mathrm{~ms}$. Though the negative $P$ waves are less readily identified, they appear to precede each QRS by a fixed interval of $190 \mathrm{~ms}$. During the gaps in recipient ventricular rhythm there is an interval of $960 \mathrm{~ms}$ without recipient QRS complexes. The RR intervals following two such gaps are 700 and $650 \mathrm{~ms}$ (Fig. 6). The likeliest explanation for this phenomenon, as illustrated in the accompanying ladder diagrams, is that the gaps are the result of atrial extrasystoles falling in the absolute refractory period of the atrioventricular conduction system and thus failing to conduct to the ventricles. These atrial extrasystoles reset the sinus node and depress its automaticity for the next few beats, thus accounting for the longer RR intervals and less obvious longer PP intervals which follow them. Because the $P$ waves could not always be clearly identified, it is possible that the first QRS complex following each gap sometimes resulted

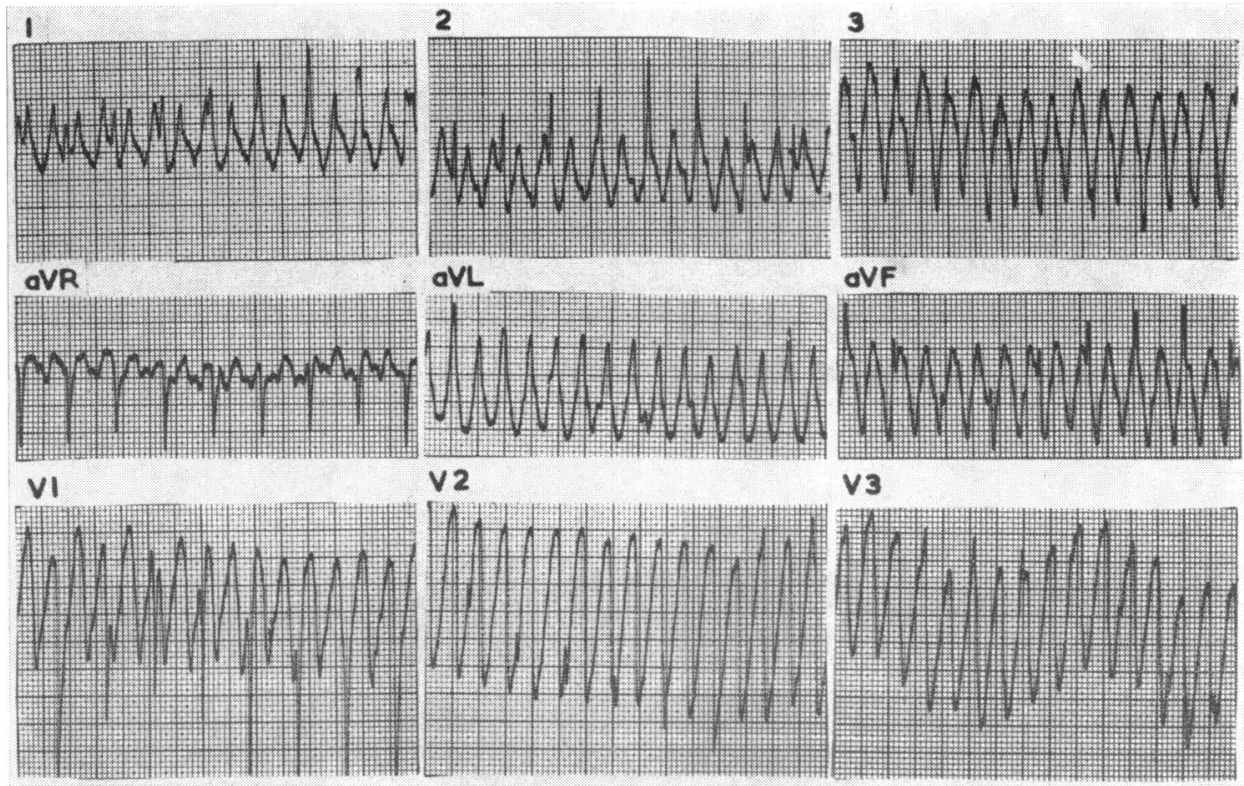

FIG. 5 Ventricular flutter in recipient heart of Case 1.

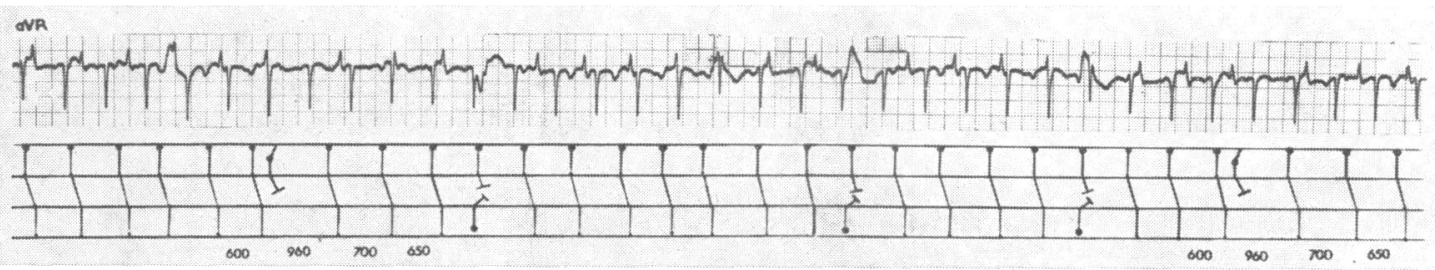

FIG. 6 Ladder diagram relates to recipient heart rhythm which manifests positive QRS complexes. Three unifocal ventricular extrasystoles are seen. Two gaps in the recipient ventricular rhythm are caused by blocked atrial extrasystoles (Case 1). 
from a supraventricular escape focus with a different $P$ wave morphology.

This patient's postoperative course was complicated by cerebral damage at the time of surgery from atheromatous embolism. This limited his mobility and he eventually died 132 days after transplantation from extensive pulmonary infarction as a result of emboli from bilateral femoral venous thromboses.

\section{Case 2}

This 47-year-old man had an aortic valve replacement in 1969 for rheumatic aortic regurgitation with grade 3 effort intolerance. The aortic prothesis had to be replaced later the same year for fungal endocarditis unresponsive to medical therapy. Between 1969 and 1972 he was much improved symptomatically, but his condition then progressively deteriorated. On physical examination, he was in sinus rhythm with obvious cardiomegaly and trivial aortic regurgitation which had been present since his second aortic valve replacement. His electrocardiogram (right-hand panel of Fig. 7) shows that he was then in sinus rhythm with first degree atrioventricular block, left atrial enlargement, left axis deviation, and left bundle-branch block. Chest $x$-ray film showed generalized massive cardiomegaly, and cardiac catheterization confirmed the clinical impression of severe biventricular cardiac failure, pulmonary hypertension, and low cardiac output; there was mild aortic regurgitation on aortography, but the left ventricle was dilated and contracted poorly on left ventricular angiography.

The patient was operated upon using the same technique as in the first patient. The donor was a 17-year-old girl who died of severe head injuries; her normal electrocardiogram is shown in the iefthand panel of Fig. 7 .
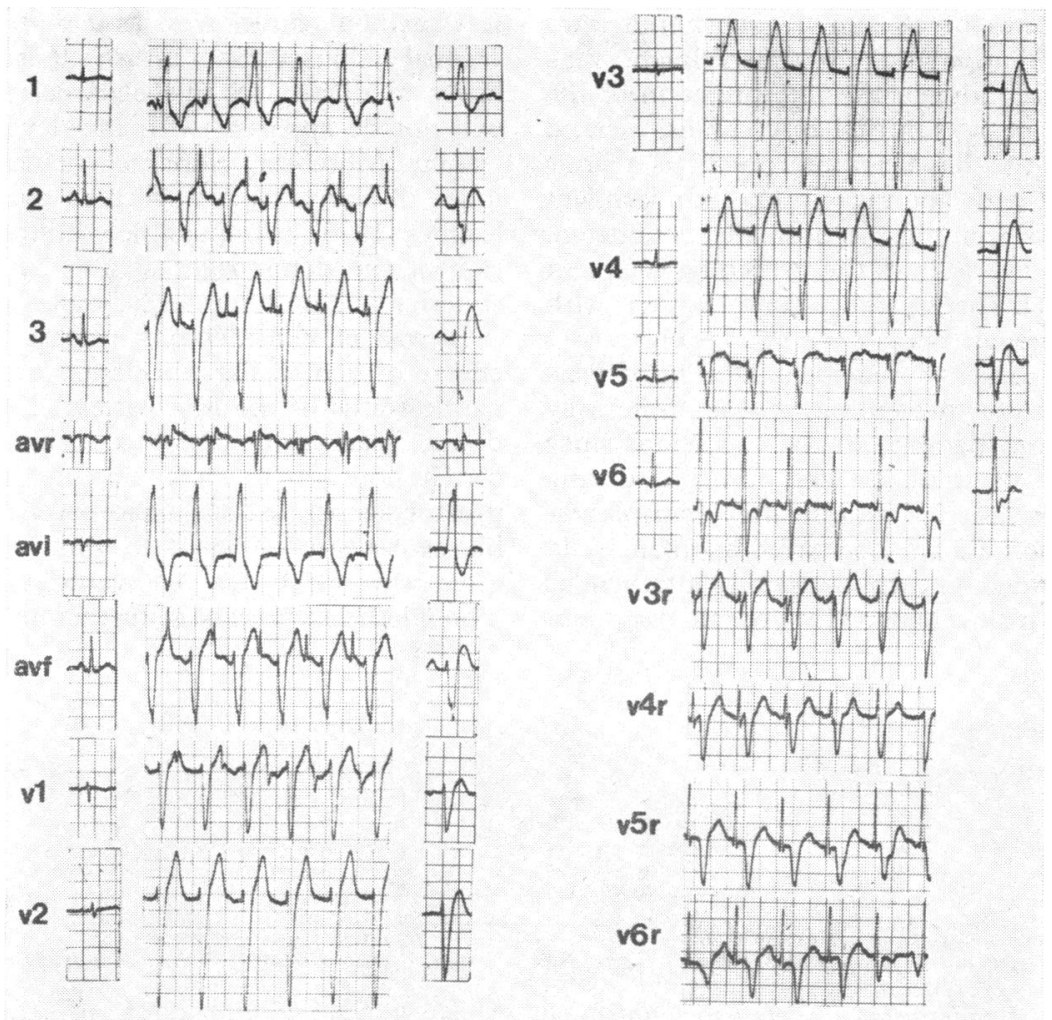

FIG. 7 Case 2: Donor electrocardiogram on left, recipient electrocardiogram on right, and postoperative electrocardiogram showing both donor and recipient complexes in centre panel. Leads $V 1$ to $V 6$ are half-standard on the recipient electrocardiogra:n. 
The postoperative electrocardiogram of this patient is illustrated in the centre panel of Fig. 7 showing both donor and recipient hearts to be in sinus rhythm. In this case also, the recipient QRS complexes are readily identified by their significant widening which was present preoperatively. The frontal plane QRS axes of the donor and recipient hearts are unchanged. Leads V5R and V6R show qR complexes in the donor heart.

Fig. 8 shows a complex arrhythmia seen in this patient six weeks after operation. The small positive QRS complexes are those of the donor heart. Most of the wider negative QRS complexes are conducted beats in the recipient heart. QRS complexes of a different morphology marked with a cross (x) have a cycle length which is a multiple of 1220 to $1240 \mathrm{~ms}$ and interfere with the recipient QRS rhythm. Another type of QRS complex marked with a circle (o) which also interferes with the recipient $Q R S$ rhythm has a cycle length which is a multiple of 1420 to $1464 \mathrm{~ms}$. Coupling intervals vary and these two ventricular rhythms are interpreted as being independent ventricular parasystolic rhythms with rates of 48 and 42 per minute, respectively, both originating in the recipient ventricle. Difficulties arise in the interpretation of the 16th recipient heart ventricular complex marked with an arrow; this has been interpreted as a sinus beat conducted with aberration. The 6 th recipient heart ventricular complex is normally conducted, though the $\mathbf{P}$ wave follows the preceding recipient heart ventricular parasystolic complex (x) with shorter RP interval.

This patient has had recurrent attacks of ventricular tachycardia, presenting in the same way as in the previous patient, on four occasions since operation. One of these attacks occurred during cardiac catheterization a month after transplantation, while the others have occurred spontaneously and have responded on each occasion to intravenous lignocaine or cardioversion. His present treatment includes quinidine gluconate, propranolol, and digoxin, and has kept him free of these arrhythmias over the past two months, though Holter tape recordings have shown intermittent ventricular parasystolic rhythms similar to those illustrated in Fig. 8. The QRS morphology of the ventricular parasystolic rhythm has been unlike that of the ventricular tachycardias. At the time of writing, he is alive and well and back at work nine months after operation.

\section{Discussion}

Changes in the electrical axis of both donor and recipient hearts in the frontal and horizontal planes are not unexpected in view of the inevitable distortion of the position of both hearts within the mediastinum. In patients with conventional heart transplants whose own hearts have been removed, postoperative rightward shifts in frontal axis are useful in diagnosing rejection (Griepp et al., 1971). In the double heart transplant situation, however, the donor heart right ventricle is under considerably less haemodynamic stress as it pumps only its own coronary sinus venous return; it is, therefore, unlikely to dilate and this electrocardiographic sign will not be apparent with the development of rejection. Moreover, right ventricular biopsy as an index of rejection, as advocated by Caves and colleagues (1973), is impossible with the surgical isolation of the donor right atrium by ligation of the superior and inferior venae cavae.

It was of considerable interest to observe the effects of ventricular fibrillation on the haemodynamic status of the first patient. Not only was his donor heart alone able to maintain a sufficient cardiac output to maintain life, but the aortic pressure generated sustained coronary perfusion of his own heart sufficient to allow its defibrillation after several hours of ventricular fibrillation. Though the occurrence of further myocardial infarc-

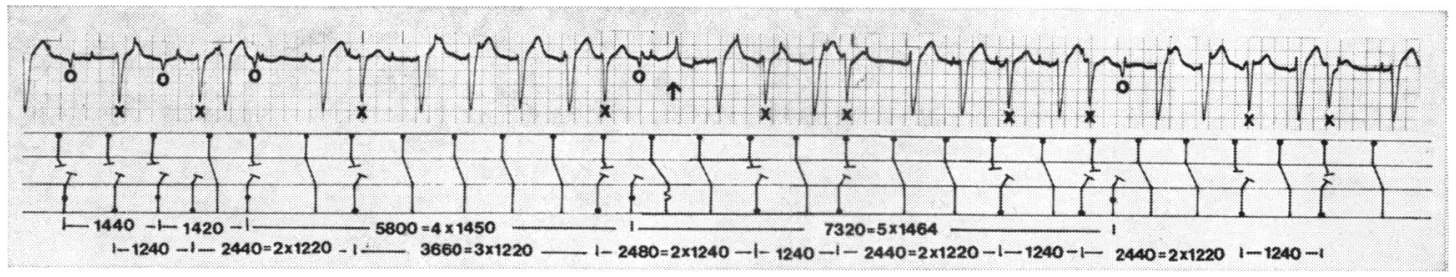

FIG. 8 Ladder diagram refers to recipient rhythm which shows nєgative QRS complexes. Two ventricular parasystolic rhythms are shown; that marked with an $(x)$ has a cycle length of 12201240 ms and that marked with an (o) has a cycle length of 1420-1464 ms. The 16th recipient ventricular complex marked with an (个) is aberrant (Case 2). 
tion in his own heart to account for the development of ventricular fibrillation could not be substantiated electrocardiographically, raised serum levels of creatine phosphokinase isoenzymes suggested this possible diagnosis. The occurrence of ventricular tachycardia and/or fibrillation in the recipient hearts of both patients after operation precipitated right heart failure. This is a possible disadvantage of left ventricular bypass transplantation, which can be overcome by simultaneously bypassing both left and right ventricles: this has since been done in a further patient operated upon at this hospital.

The interpretation of electrocardiograms in the presence of two independent rhythms presents many difficulties. Comparison of the preoperative electrocardiograms of the donor and recipient hearts helps to clarify the origin of the QRS complexes. The fusion of $P$ and $T$ waves from both hearts, however, can give rise to difficulties in interpretation. Atrial and ventricular complexes should be chosen where the timing of donor and recipient rhythms are asynchronous to assist in interpretation. Fig. 6 illustrates the difficulty in defining the suspected presence of blocked atrial extrasystoles to account for the apparent gaps in the recipient ventricular rhythm. The intact autonomic control of the recipient heart can be used to help determine in which of the two hearts the rhythm disturbances are occurring, by the use of manoeuvres or drugs which influence autonomic control of the recipient heart rate but which would be ineffective in changing the rate and rhythm of the denervated donor heart.

Ventricular parasystole is commonly seen in patients with ischaemic heart disease. The electrocardiogram in Fig. 8 shows two independent ventricular parasystolic foci, with inherent discharge rates commonly seen in this condition. The 16th recipient ventricular complex in this electrocardio- gram is interpreted as a sinus beat conducted with aberration, despite the fact that the 6th recipient ventricular complex with a shorter coupling interval after the preceding ventricular parasystolic complex is normally conducted. The suggested explanation is the presence of a gap in refractoriness of the right bundle-branch (Wellens, 1969) which results in a right bundle-branch type conduction pattern of the aberrant complex while an earlier sinus beat is normally conducted.

Interpretation of all the above electrocardiograms has been made easier by the fact that the donor heart remained in sinus rhythm throughout. Difficulties in interpretation are anticipated in the event of simultaneous rhythm disturbances in both donor and recipient hearts.

We thank the Medical Research Council, the Cape Town City Council, and the Chris Barnard Fund for financial support, and the Medical Superintendent of Groote Schuur Hospital, Dr. J. G. Burger, for permission to publish. We are grateful to Professor L. Schamroth for his assistance in reviewing this manuscript.

\section{References}

Barnard, C. N., and Losman, J. G. (1975). Left ventricular bypass. South African Medical fournal, 49, 303.

Caves, P. K., Stinson, E. B., Billingham, M. E., Rider, A. K., and Shumway, N. E. (1973). Diagnosis of human cardiac allograft rejection by serial cardiac biopsy. Fournal of Thoracic and Cardiovascular Surgery, 66, 461.

Griepp, R. B., Stinson, E. B., Dong, E., Jr., Clark, D. A., and Shumway, N. E. (1971). Acute rejection of the allografted human heart. Annals of Thoracic Surgery, 12, 113.

Wellens, H. (1969). Unusual occurrence of nonaberrant conduction in patients with atrial fibrillation and aberrant conduction. American Heart fournal, 77, 158.

Requests for reprints to Dr. B. M. Kennelly, Cardiac Clinic, Groote Schuur Hospital, Cape Town, South Africa. 